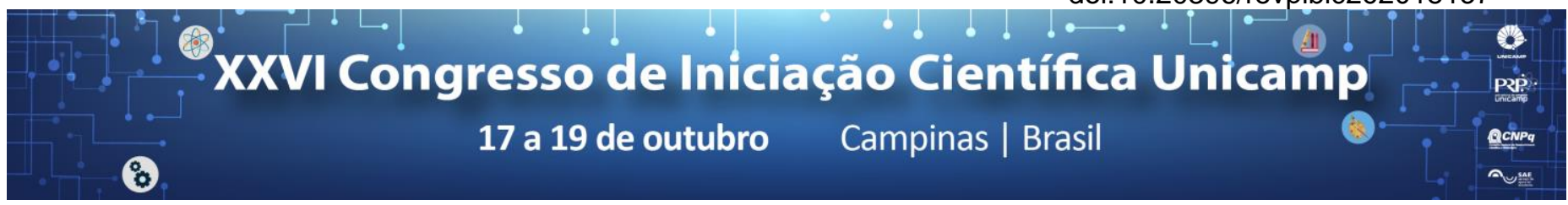

\title{
Estado nutricional, qualidade de vida e hábitos de vida em portadores da Síndrome do Intestino Irritável: um estudo caso-controle
}

\author{
Ana Paula M. Mendonça*, Luciana M. Yamashita, Esther D. Silva, Isabela Solar, Larissa A. O. Santos, Cristiane \\ K. N. V. da Cruz, Ciro G. Montes, Ana Carolina J. Vasques.
}

\section{Resumo}

A Síndrome do Intestino Irritável (SII) é a desordem gastrointestinal mais frequente na prática clínica em gastrenterologia. As alterações no hábito intestinal associadas a dor e/ou desconforto abdominal podem resultar em mudanças nos hábitos de vida, no estado nutricional e na qualidade de vida dos seus portadores. Devido ao grande desafio que a SII se tornou aos profissionais de saúde e às lacunas que ainda existem em relação ao tema, faz-se necessário estudar os portadores da SII para uma melhor compreensão do perfil clínico nutricional desses indivíduos.

\section{Palavras-chave:}

Síndrome do Intestino Irritável - Estado nutricional - Qualidade de vida

\section{Introdução}

A Síndrome do Intestino Irritável (SII) é uma desordem gastrointestinal funcional caracterizada por dor abdominal ou desconforto, irregularidade das fezes e inchaço'. Sua prevalência mundial varia de $1,1 \mathrm{a} 45 \%{ }^{1}$.

A SII está associada a pior qualidade de vida, impacto econômico adverso relacionado ao absenteísmo no trabalho e perda de produtividade, resultando gasto anual médio de bilhões de dólares com assistência médica ${ }^{2,3}$ Atualmente existem poucos estudos que correlacionam o estado nutricional, qualidade de vida $(\mathrm{QV})$ e hábitos de vida de indivíduos saudáveis com pacientes portadores da SII. Sendo assim, o objetivo do estudo é comparar o perfil de adiposidade corporal, os hábitos de vida e a qualidade de vida de indivíduos portadores da SII com um grupo controle saudável.

\section{Resultados e Discussão}

Estudo caso-controle com 30 mulheres portadoras da SII e 32 mulheres saudáveis, de 20 a 59 anos, sem doenças que afetam o estado nutricional e ingestão alimentar. A adiposidade corporal foi avaliada com o IMC, circunferências da cintura e quadril e relação cinturaquadril. A prática de atividade física foi analisada pelo IPAQ versão curta. Para avaliação dos hábitos de vida observouse etilismo, tabagismo e sonolência diurna pela Escala de Sonolência de Epworth. O Questionário de Qualidade de Vida para portadores da SII foi aplicado. CAAE: 64341316.0.0000.5404

Houve maior acúmulo de adiposidade central e periférica no grupo caso em comparação ao grupo controle $(p<0,05)$.

Os casos apresentaram maior chance de desenvolver comorbidades associadas à SII como fibromialgia $(2,8 \%$ vs $38,2 \%)$ e fadiga crônica $(2,8 \%$ vs $38,2 \%)$, transtorno têmporo mandibular $(13,9$ vs $55,9 \%)$ e dor pélvica $(5,6 \%$ vs $58,8 \%$ ) para controles e casos respectivamente, $p<0,05$ para todos. Os casos utilizam mais antidiarreicos em comparação aos controles $(p=0,008)$.

$A$ frequência no consumo de álcool é mais acentuada nos controles $(p=0,011)$. A frequência do uso do tabaco $(p=0,189)$ e sonolência diurna $(p=0,314)$ foi semelhante em ambos os grupos. Em relação à atividade física $(\mathrm{p}=0,82)$, $5,6 \%$ dos indivíduos saudáveis realizam baixa atividade física enquanto que $100 \%$ dos casos realizam atividade física moderada.
Cerca de $80 \%$ dos pacientes com a SIl excluíram algum alimento da dieta $(p<0,01)$ e o total de alimentos problemáticos $(p<0,01)$ pode variar de 7 a 21 alimentos para aqueles que possuem a síndrome.

Há pior QV geral e em todos os subdomínios nos casos em comparação aos controles $(p<0,01)$.

Tabela 1 - Comparação da qualidade de vida geral e de cada subdomínio do Questionário de Qualidade de Vida para portadores da SII, nos grupos casos e controles.

\begin{tabular}{lccc}
\hline \multicolumn{1}{c}{ Qualidade de vida } & $\begin{array}{c}\text { Controles } \\
\mathrm{n}=30\end{array}$ & $\begin{array}{c}\text { Casos } \\
\mathrm{n}=32\end{array}$ & *p \\
\hline Domínios & & & \\
Disforia & $81 \pm 23$ & $67 \pm 25$ & $<0,01$ \\
Interferência com atividade & $71 \pm 30$ & $47 \pm 24$ & $<0,01$ \\
Imagem Corporal & $71 \pm 30$ & $50 \pm 25$ & $<0,01$ \\
Preocupação com saúde & $66 \pm 32$ & $45 \pm 26$ & $<0,01$ \\
Prevenção de Alimentos & $67 \pm 31$ & $45 \pm 28$ & $<0,01$ \\
Reação Social & $84 \pm 41$ & $75 \pm 56$ & $<0,01$ \\
Sexualidade & $81 \pm 24$ & $64 \pm 34$ & $<0,01$ \\
Relacionamentos & $88 \pm 21$ & $77 \pm 27$ & 0,001 \\
Qualidade de vida geral & $76 \pm 24$ & $59 \pm 22$ & $<0,01$ \\
\hline
\end{tabular}

\section{Conclusões}

Houve maior adiposidade abdominal e generalizada associada a SII. Os hábitos de vida não diferiram entre ambos os grupos, apenas o consumo de bebida alcoólica que foi menor no grupo caso. Houve restrição alimentar na maioria dos portadores da SII (80\%) e maior presença de alimentos problemáticos em relação aos indivíduos saudáveis. Observou-se pior qualidade de vida no grupo caso.

\section{Agradecimentos}

Ao Conselho Nacional de Desenvolvimento Científico e Tecnológico (CNPq) por todo incentivo e apoio.

\footnotetext{
IIrritable bowel syndrome. Nature Reviews. Disease Primers. 2016, 2. ${ }^{2}$ Ford AC, Lacy BE, Talley NJ. Irritable bowel syndrome. N Engl J Med 2017; 376,2566-2578.

${ }^{3}$ Mullin GE, Shepherd SJ, Chander RB, Ireton-Jones C, Matarese LE. Irritable bowel syndrome: contemporary nutrition management strategies. J Parenter Enteral Nutr. 2014; 38(7), 781-799.
} 